\title{
Cross-Repressive Interaction of the Olig2 and Nkx2.2 Transcription Factors in Developing Neural Tube Associated with Formation of a Specific Physical Complex
}

\author{
Tao Sun, ${ }^{1 *}$ Hualing Dong, ${ }^{2 *}$ Lizi Wu, ${ }^{3}$ Michael Kane, ${ }^{2}$ David H. Rowitch, ${ }^{1}$ and Charles D. Stiles ${ }^{2}$ \\ Departments of ${ }^{1}$ Pediatric Oncology, ${ }^{2}$ Cancer Biology, and ${ }^{3}$ Adult Oncology, Dana-Farber Cancer Institute, Harvard Medical School, Boston, \\ Massachusetts 02115
}

In developing neural tube, the basic helix-loop-helix (bHLH) transcription factor Olig2 interacts with the homeodomain transcription factor Nkx2.2 at two distinct stages. During neuronogenesis, a cross-repressive interaction appears to establish the precise boundary between the $\mathrm{p} 3$ and $\mathrm{pMN}$ domains. At later times, a cooperative interaction is noted because Nkx 2.2 promotes maturation of oligodendrocyte progenitor cells specified by expression of Olig2. We show here that the Olig2 protein can form a physical complex with Nkx2.2 protein in mammalian cells and yeast two-hybrid trap assay. This interaction is specific because Olig2 does not bind to a biologically irrelevant homeodomain transcription factor (Nkx6.1), and $\mathrm{Nkx2.2}$ does not interact with a biologically irrelevant bHLH protein (NeuroD). Deletion mapping analysis suggests that formation of an Olig2-Nkx2.2 physical complex is insufficient for the induction of oligodendrocyte progenitors in developing spine; however, the protein-protein interaction observed might be important for the crossrepressive interaction between Olig2 and Nkx2.2 that helps to establish the pMN-p3 boundary in the developing spinal cord.

Key words: Nkx2.2; Olig2; homeodomain; bHLH; neural tube; neuronal subtype specification; oligodendrocyte; interneuron

\section{Introduction}

During vertebrate CNS development, coincident spatialtemporal expression of transcription factors is thought to comprise a combinatorial code that specifies formation of dedicated neural progenitor cells and emerging waves of specialized neuronal and glial progeny (Jessell, 2000; Bertrand et al., 2002). Two structurally distinct types of transcription factors are principal components of this code. Homeodomain (HD) transcription factors are defined by a 60 amino acid DNA-binding motif that forms a highly conserved helix-turn-helix structure. Within the NK-2 subclass of HD transcription factors, Nkx2.2 defines the $\mathrm{p} 3$ progenitor domain and is specifically required for production of V3 interneurons (INs) that express Sim 1 (Briscoe et al., 1999) and maturation of oligodendrocytes (Qi et al., 2001).

Transcription factors with basic helix-loop-helix (bHLH) domains have fundamental roles during neuronal and glial production (Gowan et al., 2001; Bertrand et al., 2002). The basic domain is required for interaction with specific DNA elements, whereas

\footnotetext{
Received April 28, 2003; revised Aug. 26, 2003; accepted Aug. 28, 2003.

T.S. thanks the National Multiple Sclerosis Society for an advanced postdoctoral fellowship. D.H.R. is a Claudia Adams Barr Investigator and Harry Weaver Neuroscience Scholar of the National Multiple Sclerosis Society. These studies were funded by National Institute of Neurological Diseases and Stroke Grant NS4051 (D.H.R.), National Institute of Child Health and Development Grant HD24296 (C.D.S.), and the Dana-Farber/Mahoney Center for Neuro-Oncology.

*T. S. and H. D. contributed equally to this work.

Correspondence should be addressed to either of the following: David H. Rowitch, Department of Pediatric Oncology, Dana-Farber Cancer Institute, Harvard Medical School, 44 Binney Street, Boston, MA 02115, E-mail: david_rowitch@dfci.harvard.edu; or Charles D. Stiles, Department of Cancer Biology, Dana-Farber Cancer Institute, Harvard Medical School, 44 Binney Street, Boston, MA 02115, E-mail: charles_stiles@dfci.harvard.edu. Copyright $\odot 2003$ Society for Neuroscience $\quad$ 0270-6474/03/239547-10\$15.00/0
}

the helix-loop-helix motif mediates homodimerization or heterodimerization, which is required for biological activity. Cell type-specific bHLH proteins such as those involved in spinal cord patterning typically do not form homodimers. Rather they interact with E proteins (E12, E47, E2-2, and HEB), a family of bHLH factors that are broadly expressed (Murre et al., 1989; Zhuang et al., 1998). Among the cell type-specific bHLH transcription factors expressed in the developing spinal cord, function of Olig2 is required for motor neuron and oligodendrocyte development in the pMN domain (Lu et al., 2002; Park et al., 2002; Takebayashi et al., 2002a; Zhou and Anderson, 2002).

Gain-of-function and loss-of-function genetic analyses suggest that Olig2 interacts with $\mathrm{Nkx} 2.2$ at two distinct stages in developing spine. During neuronogenesis, a cross-repressive interaction between Olig2 and Nkx2.2 contributes to formation of the boundary between the $\mathrm{p} 3$ and $\mathrm{pMN}$ domains in the ventral neural tube (Mizuguchi et al., 2001; Novitch et al., 2001; Sun et al., 2001; Zhou et al., 2001). At later times, a cooperative interaction is noted. Delayed differentiation of oligodendrocyte precursors in Nkx2.2 null mice indicates that Nkx2.2 promotes maturation of oligodendrocyte progenitor cells specified by expression of Olig2 (Qi et al., 2001). In chick neural tube, this cooperative interaction can be visualized by ectopic formation of oligodendrocytes after co-electroporation of Olig2 and Nkx2.2 (Sun et al., 2001; Zhou et al., 2001).

The biochemical mechanisms underlying cooperative and cross-repressive interactions between Olig2 and Nkx2.2 are poorly defined. In principle, these structurally distinct transcription factors should function independently of each other within 
the cell nucleus by regulating the expression of distinct gene sets. In this study, however, we show that Nkx2.2 and Olig2 physically interact with each other to form a specific intranuclear complex. The domains required for this biochemical interaction are not sufficient for oligodendrocyte formation during CNS development but they are sufficient for a cross-repressive interaction.

\section{Materials and Methods}

Plasmid constructs. The full-length mouse Olig2 cDNA was subcloned into a pcDNA3.1 expression vector (Invitrogen, San Diego, CA) with the cytomegalovirus (CMV) promoter and an in-frame Myc- or V5-tag at the $\mathrm{C}$ terminus. A series of deletions of Olig2 were amplified by PCR using the full-length mouse Olig2 cDNA as template and then subcloned into a pRK5 vector (gift from Dr. Anthony Lanahan, Johns Hopkins Medical Institute, Baltimore, MD) with the CMV promoter and an inframe Myc-tag at the $\mathrm{N}$ terminus.

The full-length mouse $N k x 2.2$ cDNA was subcloned into a pFLAGCMV-2 expression vector (Sigma, St. Louis, MO) with the CMV promoter and an in-frame Flag-tag at the $\mathrm{N}$ terminus, or pcDNA3.1 expression vector (Invitrogen) with an in-frame Myc-tag at the $\mathrm{C}$ terminus. A series of deletions of $N k \times 2.2$ were amplified by PCR using the full-length mouse $N k x 2.2 \mathrm{cDNA}$ as template and then subcloned into a pRK5 vector with the CMV promoter and an in-frame hemagglutinin (HA)-tag at the $\mathrm{N}$ terminus. Similarly, the full-length mouse NeuroD cDNA was subcloned into pRK5 expression vector with the $\mathrm{N}$ terminus Myc-tag. The full-length mouse Nkx6.1 (a gift from Dr. M. German, University of California San Francisco) and E12 cDNA were subcloned into a pRK5 vector with the $\mathrm{N}$ terminus HA-tag.

Yeast two-hybrid assay. Bait vector harboring full or partial Olig2 cDNA and target vector harboring full or partial $N k \times 2.2$ cDNA were cotransformed into yeast (pJ69-4A or Y190 strains) using a standard protocol (Clontech, Cambridge, UK). Full-length Olig2 and its bHLH domain were subcloned into a pAS2-1 vector (Clontech) or the pPC97 vector, respectively, with the in-frame Gal4 DNA binding domain at the $\mathrm{N}$ terminus. Full-length $N k \times 2.2$ and a series of $N k \times 2.2$ deletions were subcloned into the pACT2 vector (Clontech) or the pPC86 vector, respectively, with the in-frame Gal4 DNA binding domain at the $\mathrm{N}$ terminus. The PC97 and PC86 vectors were gifts from Dr. Anthony Lanahan (John Hopkins Medical Institute). Colonies displaying two-hybrid interactions were visualized on Leu-Trp-His-plates, as well as on the more stringent $\beta$-galactosidase filter assay.

Cell culture and transient transfection. Cos7 cells were cultured in DMEM with $10 \%$ fetal bovine serum and penicillin (100 U/ml)-streptomycin $(50 \mu \mathrm{g} / \mathrm{ml})$ at $37^{\circ} \mathrm{C}$ with $5 \% \mathrm{CO}_{2}$ to a density of $2-3 \times 10^{6}$ cells per $100 \mathrm{~mm}$ plate at the time of transfection. Transfections were performed for $1-4 \mathrm{hr}$ by using the Lipofectamine 2000 reagent (Invitrogen) according to the manufacturer's protocol. Equal amounts of plasmid DNA were used for cotransfection. For immunostaining experiments, Cos7 cells were plated on coverslips in the 24-well plates.

Western blot analysis and co-immunoprecipitation. Cos7 cells were washed in PBS 24-40 hr after transfection, and protein lysates were harvested in buffer $\mathrm{H}$ ( $50 \mathrm{~mm}$ Tris, $\mathrm{pH} 7.5$, with $5 \mathrm{~mm}$ EDTA, $0.5 \mathrm{~mm}$ $\mathrm{Na}_{3} \mathrm{VO}_{3}, 5 \mathrm{~mm} \mathrm{NaF}$, adding fresh $0.2 \%$ NP-40, 1 mм DTT, 1 mм PMSF, $1 \mu \mathrm{g} / \mu \mathrm{l}$ aprotinin, $1 \mu \mathrm{g} / \mu \mathrm{l}$ leupeptin before use) and rotated at $4^{\circ} \mathrm{C}$ for 20 min; 0.11 volume of $170 \mathrm{~mm} \mathrm{NaCl}$ was then added to extract the nuclear protein. The lysates were further rotated at $4^{\circ} \mathrm{C}$ for $20 \mathrm{~min}$, centrifuged at $13,000 \mathrm{rpm}$ for $10 \mathrm{~min}$ at $4^{\circ} \mathrm{C}$, and finally frozen at $-80^{\circ} \mathrm{C}$ until they were used.

For each co-immunoprecipitation (Co-IP), 1-2 $\mu \mathrm{g}$ of monoclonal anti-Myc (Santa Cruz Biotechnology, Santa Cruz, CA) or anti-Flag (Sigma) antibodies were mixed (1:1) with $100 \mu$ l of protein G Sepharose beads (Amersham Biosciences, Arlington Heights, IL), preswelled in buffer $\mathrm{H} ; 600 \mu \mathrm{l}$ of buffer $\mathrm{H}$ was then added. The antibody-protein-G bead mixture was rotated at $4^{\circ} \mathrm{C}$ for $1 \mathrm{hr}$ and then centrifuged at 13,000 $\mathrm{rpm}$. Between 100 and $400 \mu \mathrm{l}$ of cell lysate was added in the mixture, and Buffer $\mathrm{H}$ was added to the final volume of $600-700 \mu$ l. The mixture was rotated at $4^{\circ} \mathrm{C}$ for $1-2 \mathrm{hr}$. Precipitates were then washed twice with $1 \mathrm{ml}$ of ice-cold buffer $\mathrm{H}$, resuspended in $120 \mu \mathrm{l} 1.5 \times$ SDS sampling buffer, and boiled for $5 \mathrm{~min}$. After centrifugation, the supernatant was size fractionated on a 12 or $15 \%$ SDS-PAGE gel and transferred to a polyvinylidene difluoride membrane (Millipore, Bedford, MA).

The Western blot analysis was performed using monoclonal or polyclonal anti-Myc antibodies (1:500; Santa Cruz Biotechnology), monoclonal anti-HA antibodies (1:5000; Babco, Richmond, CA), monoclonal anti-Flag antibodies (1:800; Sigma), and monoclonal anti-Nkx2.2 antibodies [1:500; Developmental Studies Hybridoma Bank (DSHB), Iowa City, IA]. The secondary alkaline phosphatase-conjugated anti-mouse or

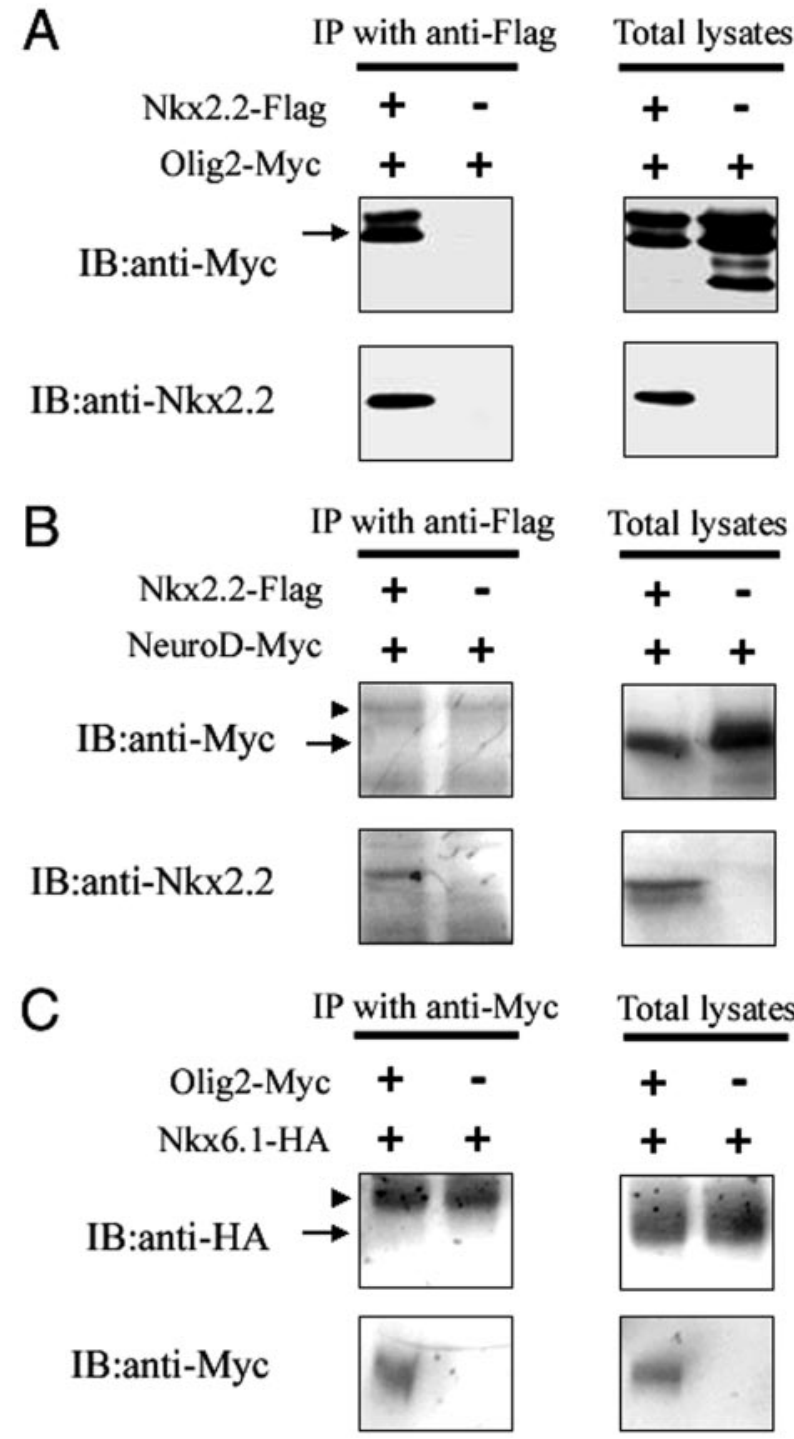

Figure 1. Specific physical interaction between Nkx2.2 and 0lig2. A, Cos7 cells were cotransfected with expression constructs encoding Flag-tagged Nkx2.2 and Myc-Tagged Olig2. Western blots from anti-Flag immunoprecipitates (IP) and also total cell lysates were immunoblotted (IB) with anti-Myc and anti-Nkx2.2 antibodies. Olig2 was immunoprecipitated by full-

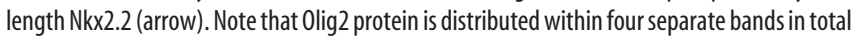
cell lysates. Two of these bands may reflect alternate translation initiation sites in the 0lig2 cDNA sequence (Lu et al., 2000), and the other two may reflect degradation-post-translational modification products. $B$, Cos7 cells were cotransfected with expression constructs encoding Flag-tagged Nkx2.2 and Myc-Tagged NeuroD. Western blots from anti-Flag immunoprecipitates and also from total cell lysates were immunoblotted with anti-Myc and anti-Nkx2.2 antibodies. NeuroD was not immunoprecipitated by the full-length Nkx2.2 (arrow indicates the expected NeuroD band, and arrowhead indicates nonspecific lgG band.). C, Cos7 cells were cotransfected with expression constructs encoding Myc-tagged Olig2 and HA-Tagged Nkx6.1. Western blots from anti-Myc immunoprecipitates and also from total cell lysates were immunoblotted with anti-HA and anti-Myc antibodies. Olig2 fails to coimmunoprecipitate with Nkx6.1 (arrow indicates the expected Nkx6.1 band, and arrowhead indicates nonspecific lgG band). 


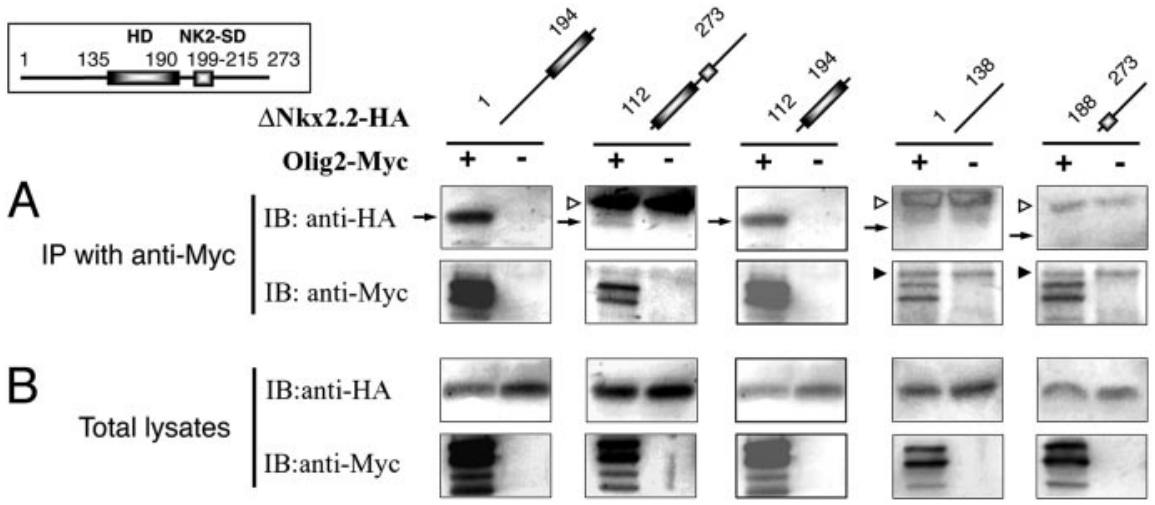

Figure 2. The homeodomain of Nkx2.2 is both necessary and sufficient for physical interaction with 0lig2. A, Cos7 cells were cotransfected with expression constructs encoding Myc-tagged full-length Olig2 and HA-tagged deletions of Nkx2.2. Western blots of the anti-Myc coimmunoprecipitates were immunoblotted with anti-HA and anti-Myc antibodies. The scheme of fulllength $\mathrm{Nkx2.2}$ is shown on the top left, and schemes of Nkx2.2 deletions are shown above each pair of lanes. Arrows indicate the predicted positions of coimmunoprecipitated Nkx2.2 deletion fragments. Nonspecific bands of $\mathrm{g} G$ heavy chain and light chain are indicated by black arrowheads and white arrowheads, respectively. As shown, deletion mutations of Nkx2.2 containing HD domains can be coimmunoprecipitated by the full-length 0 lig2, whereas deletion mutants that lack HD cannot be coimmunoprecipitated. $B$, Western blots of total cell lysates were immunoblotted with anti-HA and anti-Myc antibodies.

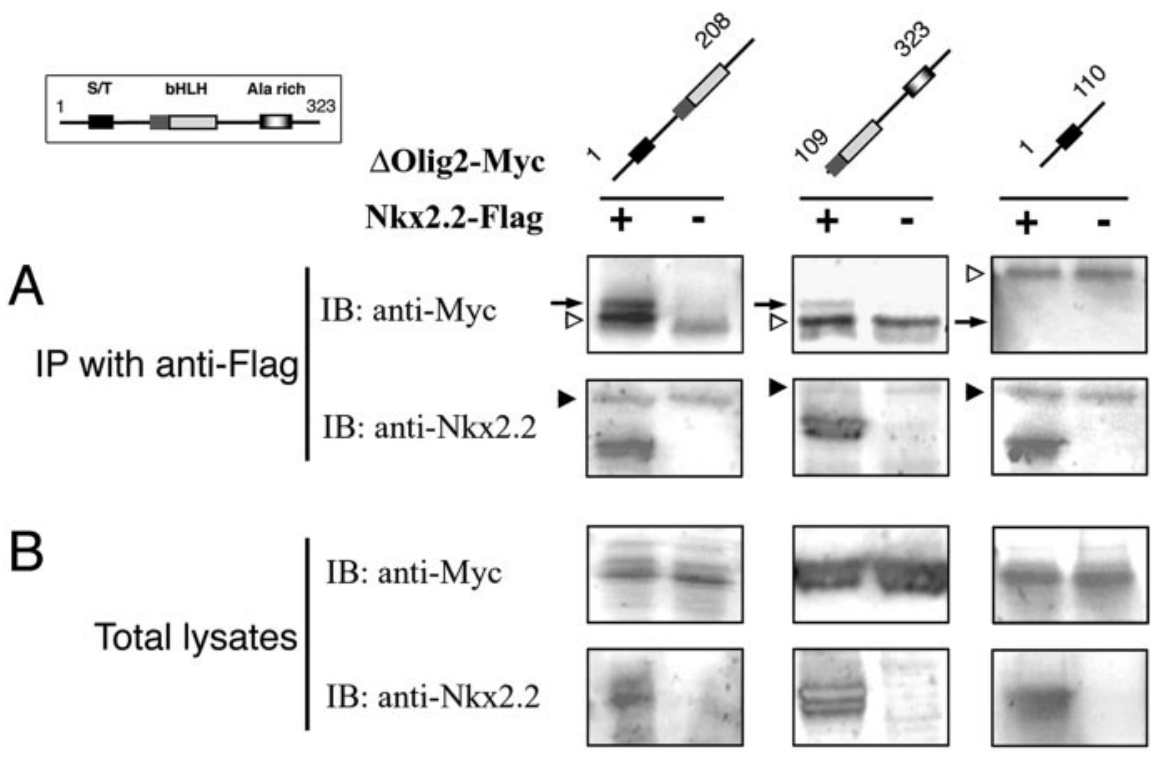

Figure 3. The bHLH domain of Olig2 is both necessary and sufficient for physical interaction with Nkx2.2. A, Full-length and deletion mutations of 0 lig2 (all Myc tagged) were cotransfected with the Flag-tagged full-length Nkx2.2 into Cos7 cells. Anti-Flag immunoprecipitates were immunoblotted with anti-Flag and anti-Myc antibodies as in Figure 2. The scheme of full-length 0 lig 2 is shown on the top left, and schemes of Olig2 deletions are shown on the top. The predicted positions of coimmunoprecipitated Olig2 deletion fragments are indicated by arrow. Nonspecific bands of IgG heavy chain and light chain are indicated by black arrowheads and white arrowheads, respectively. As shown, 0 lig2 mutations containing bHLH domains are coimmunoprecipitated by the full-length Nkx2.2. B, Western blots of total cell lysates were immunoblotted with anti-Myc and anti-Nkx2.2 antibodies. The doublet of Nkx2.2 bands shown here may reflect partial degradation of the Flag-tagged Nkx2.2 during handling or a distortion of thick gel during transferring on the membrane.

rabbit antibodies (1:5000; Promega, Madison, WI) were used and reacted with AttoPhos Fluorescent Substrate (1:25; Promega). Proteins were visualized with STORM 860 Scanner (Molecular Dynamics, Sunnyvale, CA).

Immunohistochemistry and in situ hybridization. Transfected cells were washed briefly in cold PBS, fixed in $4 \%$ PFA in PBS, pH 7.0, at $4^{\circ} \mathrm{C}$ for 10 min, and then washed with PBS. Cells were labeled with the first antibodies in PBS with $0.1 \%$ Triton X-100 and $10 \%$ normal goat serum at room temperature for $1 \mathrm{hr}$. After they were washed with PBS, cells were labeled with the second antibodies at room temperature for $1 \mathrm{hr}$ followed by $4^{\prime}, 6^{\prime}$-diamidino-2-phenylindole (DAPI) staining to visualize the nuclei. For immunohistochemistry on sections, chick embryos were fixed in $4 \%$
PFA in PBS and cryosectioned. The following primary antibodies were used at the indicated dilutions: murine Nkx2.2 (1:500; DSHB), rat Nkx2.2 (1:5000; gift from T. Jessell, Columbia University, New York), Olig2 (1:4000), Myc (1: 200; Invitrogen), and HA (1:5000; Babco). Secondary antibodies used were anti-mouse IgG FITC (1:100; Jackson ImmunoResearch, West Grove, PA), and anti-rabbit IgG Cy3 (1:200, Jackson ImmunoResearch). Photomicrographic images were collected on a Nikon E600 microscope and RT Monochrome SPOT digital camera.

In situ hybridization was performed using a modified protocol (Ma et al., 1997), and detailed instructions are available on request. Digoxygenin (DIG)-labeled antisense mRNA probes for $c \operatorname{Sox} 10$ and $c \operatorname{Sim} 1$ were prepared by in vitro transcription.

Electroporation in ovo. The chick neural tubes at Hamburger and Hamilton $(\mathrm{HH})$ stage $10-12$ were electroporated unilaterally (five 50 msec pulses at $25 \mathrm{~V})$ with cDNAs $(3 \mu \mathrm{g} / \mu \mathrm{l})$ using an ECM830 electro-squareporator (BTX Inc.). For co-electroporation, equal amounts of plasmid DNA ( $3 \mu \mathrm{g} / \mu \mathrm{l}$ each) were mixed before electroporation. Embryos were analyzed after $48 \mathrm{hr}$ incubation (HH stages 21-23). At least 10 electroporated embryos were analyzed for each plasmid DNA construct. Results documented in the figures are representative of at least five embryos. Expression constructs driven by the CMV promoter were used in all experiments. The advantage of these CMV-driven constructs is that fate changes are confined to the primary targets of the electroporation. By contrast, replicationcompetent avian retrovirus expression vectors allow secondary infection of neural progenitors (Morgan and Fekete, 1996). Note that gene expression driven by CMV vectors is attenuated in vivo 2-3 d after the electroporation.

\section{Results Specific physical interaction between Nkx2.2 and Olig2}

To identify co-regulator proteins that might interact with Olig2 and $N k \times 2.2$, we performed a series of antibody pull-down experiments. Cos7 cells were transfected or cotransfected with expression constructs encoding Flag-tagged Nkx2.2 and Myctagged Olig2. Somewhat to our surprise, these pull-down experiments showed that Olig2 interacts with $\mathrm{Nkx} 2.2$ when the two transcription factors are coexpressed (Fig. 1A).

The bHLH transcription factor NeuroD is expressed in postmitotic neurons in the CNS (Lee et al., 1995; Roztocil et al., 1997) and has no known cooperative or cross-repressive interactions with $\mathrm{Nkx2.2}$. As shown in Figure $1 B$, Nkx2.2 does not interact with this biologically irrelevant bHLH protein. The HD transcription factor Nkx6.1 is broadly expressed in the ventral region of developing spine and thus overlaps the expression domain of Olig2 at early times in development as does Nkx2.2 (Sander et al., 2000; Novitch et al., 2001); however, Nkx6.1 has no known biological interactions with Olig2. As shown in Figure 1C, Olig2 does not coprecipitate with Nkx6.1. Together these results suggest that 
Table 1. Olig2 and Nkx2.2 interact via bHLH and HD domains in yeast two-hybrid assays

\begin{tabular}{llll}
\hline Bait & Target & L-T-A growth & $\beta$-Gal assay \\
\hline Olig2 & Target vector & - & - \\
Bait vector & Nkx2.2 full length & - & - \\
Olig2 full length & Nkx2.2 full length & +++ & $+/-$ \\
Olig2 bHLH domain & Nkx2.2 HD domain & +++ & ++ \\
\hline
\end{tabular}

Full length Olig2 or the bHLH domain (93-170 aa) were fused with Gal4 DNA binding domains in the pAS2 vector. Full-length Nkx2.2 or the Nkx2.2 HD (112-194 aa) were fused to Gal4 activation domains in the pACT2 vector. The fusion constructs were cotransformed into the Y190 yeast strain. Interactions were assayed with triple minus leucine, tryptophan, adenosine (L-T-A) plates and $\beta$-gal assays were performed with grown colonies. Colony growth and blue color in $\beta$-gal assays were evaluated subjectively: +++ , Strong interaction; - , no interaction ,+++ , and $+/-$ are intermediate interactions.
A

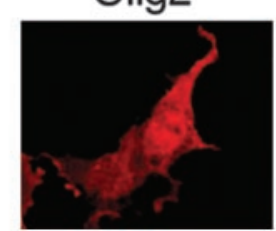

B

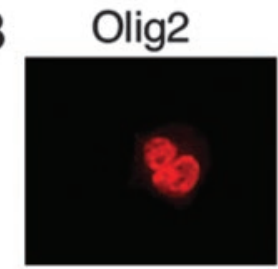

C

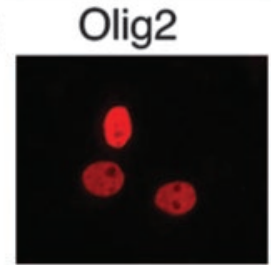

D

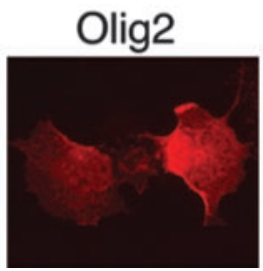

Nkx2.2

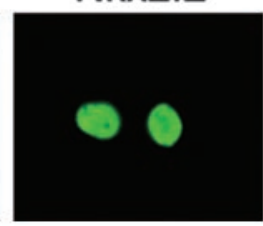

E12

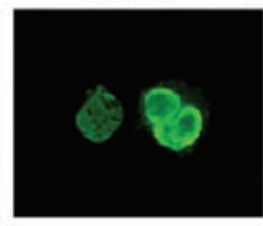

Nkx2.2

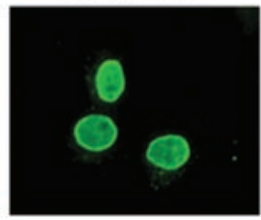

Nkx6.1

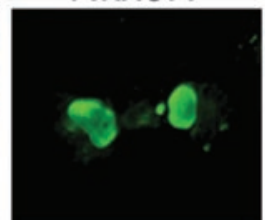

Nkx6.1

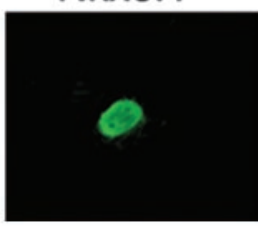

merge

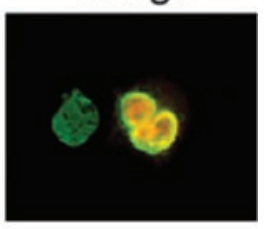

merge

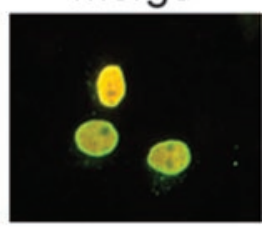

merge

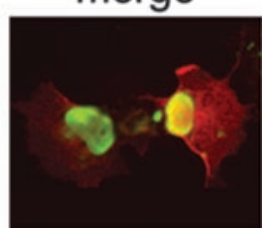

Figure 4. Olig2 and Nkx2.2 interact within mammalian cells. A, Full-length Olig2, Nkx2.2, and HA-tagged Nkx6.1 were transfected into Cos7 cells individually. Transfected cells were labeled with anti-0lig2 antibodies (red), anti-Nkx2.2 antibodies (green), and anti-HA antibodies (green). Olig2 is localized in the cytoplasm and nucleus, whereas Nkx2.2 and Nkx6.1 are within the nuclei. $B$, Full-length Olig2 and $\mathrm{HA}$-tagged $E 12$ were cotransfected into $\operatorname{Cos} 7$ cells. Transfected cells were labeled with anti-0lig2 antibodies (red) and anti-HA antibodies to visualize E12 (green). Olig2 is recruited into nuclei by cotransfection with E12 (yellow in overlay).C, Full-length Olig2 and Nkx2.2 were cotransfected into Cos7 cells. Transfected cells were labeled with anti-0lig2 antibodies (red) and anti-Nkx2.2 antibodies (green). 0lig2 is localized into nuclei by cotransfection with Nkx2.2 (yellow in overlay). D, Full-length Olig2 and Nkx6.1 were cotransfected into Cos7 cells. Transfected cells were labeled with anti-0lig2 antibodies (red) and anti-HA antibodies to visualize Nkx6.1 (green). Nkx6.1 fails to bring Olig2 into nuclei in cotransfected cells.

Olig2 interacts with $\mathrm{Nkx} 2.2$, either directly or indirectly, to form a protein complex and this interaction is not merely adventitious.

Physical interactions are mediated by the Nkx2.2

homeodomain and the Olig2 bHLH domain

To identify regions of $\mathrm{Nkx} 2.2$ and Olig2 that are required for physical interaction, we constructed a series of deletion mutants of Nkx2.2 with HA-tag and Olig2 with Myc-tag. To identify regions of Nkx2.2 that are necessary for physical interaction with Olig2, we performed antibody pull-down experiments using fulllength Olig2-Myc and the panel of Nkx2.2 deletion mutants. Full-length Nkx2.2 contains a homeodomain and an NK-2specific domain (NK2-SD) (Fig. 2). As shown, Nkx2.2 deletions without homeodomains do not interact with Olig2. Conversely, Nkx2.2 deletions that contain an HD are coimmunoprecipitated with Olig2 (Fig. 2). Thus, for physical interaction with Olig2, the homeodomain of Nkx2.2 is both necessary and sufficient.

In reciprocal experiments, we defined the regions of Olig2 that are required to form a complex with full-length $\mathrm{Nkx} 2.2$. As shown in Figure 3, salient structural features of Olig2 include the bHLH domain, a cluster of contiguous serine-threonine (S/T) residues in the $\mathrm{N}$-terminal region, and an alanine-rich region (Ala) at the $\mathrm{C}$ terminus. In repeated attempts, we were unable to express constructs encoding the Olig2 bHLH domain alone at levels sufficient to detect by immune precipitation (see Fig. 8); however, combinatorial cotransfections summarized in Figure 3 show that the $\mathrm{C}$ - and $\mathrm{N}$-terminal domains of Olig2 are individually dispensable to formation of a stable complex with $\mathrm{Nkx} 2.2$. Conversely the bHLH domain is essential and possibly sufficient.

Olig2 and Nkx2.2 interact in yeast two-hybrid assays

Antibody pull-down experiments of the sort shown in Figures 1-3 are vulnerable to artifact. Depending on conditions of salt and $\mathrm{pH}$ used to wash the Sepharose beads, protein-protein interactions can be made to appear or disappear. Accordingly we looked for evidence of a physical interaction between Olig proteins and Nkx2.2 in living cells. As shown in Table 1, Olig proteins and Nkx2.2 interact in yeast two-hybrid assays. As was noted in antibody pull-down experiments, the only protein domains required for this interaction are the bHLH domain of Olig2 and the homeodomain of Nkx2.2. In fact, the two-hybrid interaction is more robust when the sequences flanking the bHLH and homeodomain are deleted (Table 1).

\section{Olig2 and Nkx2.2 interact within mammalian cells}

Another indication of an Olig2-Nkx2.2 interaction in living cells is suggested by an interesting relocalization of Olig2 protein in Cos7 cell transfection experiments. Within the ventral neural tube of developing mice and chicks, endogenous Olig2 protein appears to be sequestered within the nucleus as expected of a bHLH transcription factor (Novitch et al., 2001; Sun et al., 2001; Zhou et al., 2001; Takebayashi et al., 2002b). As shown in Figure $4 A$, however, Olig2 protein is sequestered within the cytosol and perinuclear region of Cos7 cells when it is expressed by itself. Coexpression with a class E bHLH partner protein (E12) allows translocation of Olig2 to the nucleus (Fig. $4 B$ ); however, as shown, coexpression with Nkx2.2 will also relocalize Olig2 to the nucleus (Fig. 4C). By contrast, coexpression with Nkx6.1 does not relocalize Olig2 to the nucleus (Fig. 4D).

To map the domains of Olig2 and Nkx2.2 that mediate this response in mammalian cells, we first examined the location of deletion mutants in single transfection assays. As shown in Figure $5 A$, the default location of $\mathrm{Nkx} 2.2$ is within the nucleus of Cos7 cells. The HD domain is both necessary and sufficient for this nuclear localization. Conversely, the default location of Olig2 is within the cytosol-perinuclear region of Cos7 cells, and the entire protein sequence is required to maintain this position. Deletion of any material to the $\mathrm{N}$ - or C-terminal side of the bHLH domain allows Olig2 to gain access to the nucleus (Fig. 5B) (see Discussion). 


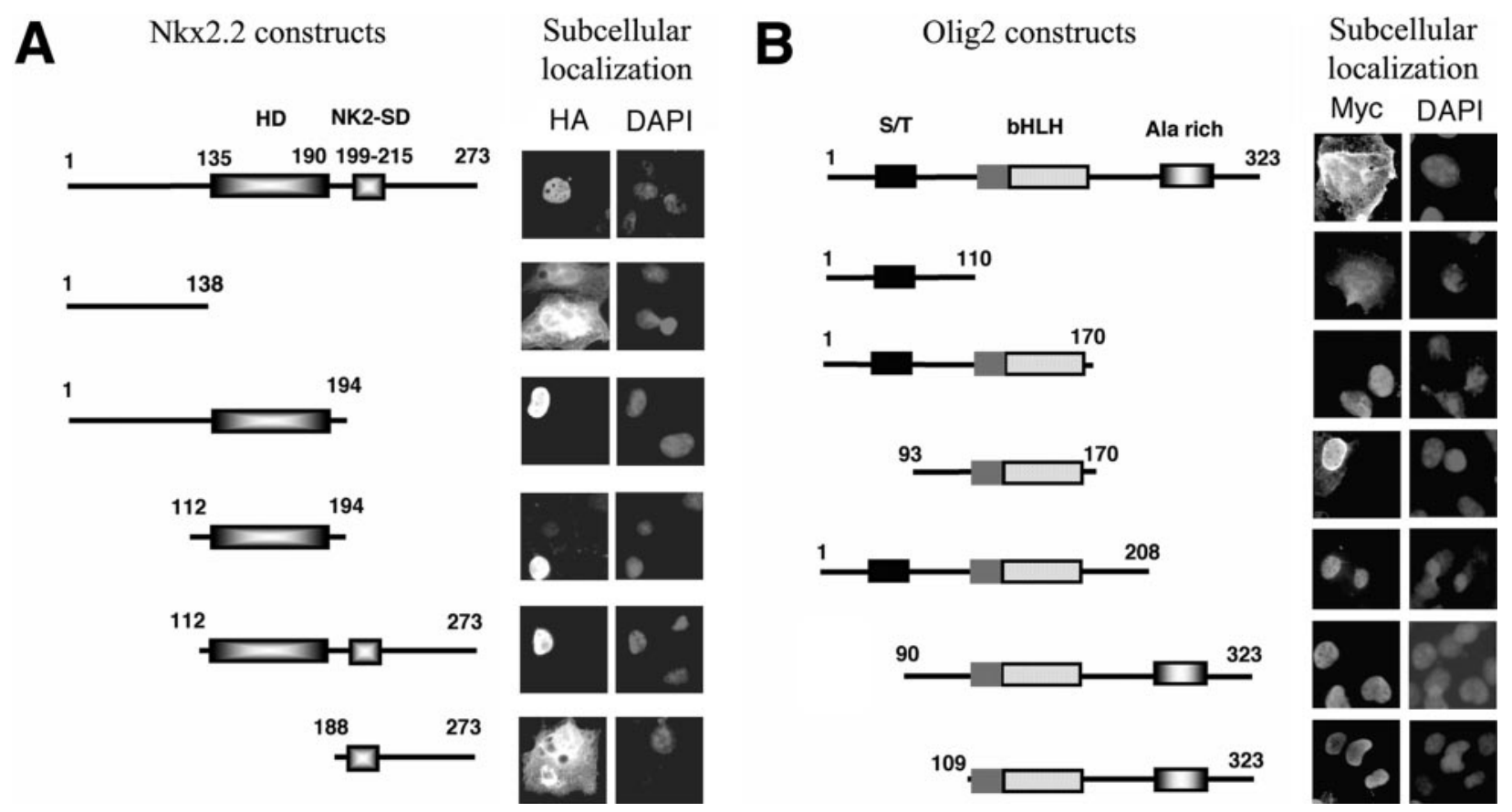

Figure 5. Mapping Nkx2.2 and Olig2 domains that specify subcellular localization of the wild-type proteins. A, Schemes of a series of Nkx2.2 deletion mutations. Full-length Nkx2.2 with Flag tag and deletion mutations with HA tag are illustrated schematically according to amino acid content. The HD (135-190 aa) and the NK2-SD (199-215 aa) are labeled separately. Subcellular localizations of each deletion fragment in transfected $\operatorname{Cos} 7$ cells were established by using anti-HA antibodies and DAPI to visualize nuclei. As shown, the HD domain of Nkx2.2 is essential for nuclear localization. B, Schemes of a series of Olig2 deletion mutations. The full-length Olig2 and each deletion mutation with Myc tag are illustrated according to amino acid content. The bHLH domain, S/T, and Ala-rich domain are labeled separately. Subcellular localization of each deletion fragment in transfected Cos7 cells was visualized by using anti-Myc antibodies and DAPI to visualize nuclei. Full-length 0 lig2 is localized in the cytoplasm and the nucleus, whereas all deletions of Olig2 with bHLH domains are localized in nuclei.

To define the regions of Nkx2.2 essential for recruiting fulllength Olig2 into the nucleus, we cotransfected full-length Olig2 and $N k \times 2.2$ deletion mutants into Cos7 cells. As shown in Figure 6, Nkx2.2 deletion mutants that lack HDs do not affect Olig2 localization; however, $N k \times 2.2$ deletion mutants that contain only HDs likewise fail to recruit Olig2 into the nucleus (Fig. 6). The $\Delta \mathrm{Nkx} 2.2(112-273 \mathrm{aa})$ mutant, which contains both HD and NK2-specific domains, mimics full-length Nkx2.2 in recruiting Olig2 into the nucleus (Fig. 6). Thus, in this mammalian cellbased assay, the Nkx2.2 homeodomain is essential but not sufficient for interaction with Olig2. The NK2-specific domain contributes to the interaction.

\section{Interaction domains of Olig2 and Nkx2.2 are not sufficient} for cooperative biological function

Data shown to this point indicate that Olig2 and Nkx2.2 form a specific physical complex in vitro and in vivo. To define the role of this interaction in their cooperative function to form oligodendrocytes, we electroporated (singly or together) a panel of Olig2 and $N k \times 2.2$ expression vectors into developing chick neural tubes. In confirmation of previous studies (Sun et al., 2001; Zhou et al., 2001), neither Olig2 nor Nkx2.2 alone promotes oligodendrocyte development in chick neural tube (Fig. $7 A, B$ ), whereas the combination stimulates ectopic formation of oligodendrocyte progenitor cells marked by Sox 10 (Fig. 7C). Deletion of any material flanking the HD domain ablates the ability of Nkx2.2 to cooperate with Olig2 in this biological response. Conversely, deletion of any material outside the bHLH domain ablates the ability of Olig2 to cooperate with Nkx2.2 (Fig. 7D-F) (data not shown). Thus the domains of Olig2 and Nkx2.2 that are sufficient to promote a physical interaction of the two proteins are not sufficient to promote this developmental response. The physical interaction of these two proteins may be irrelevant to their cooperative function in promoting formation of oligodendrocytes (see Discussion). Alternatively, other subregions of both proteins are required to initiate formation of oligodendrocytes in developing neural tube.

\section{Interaction domains of Olig2 and Nkx2.2 are sufficient for cross-repressive function}

Previous studies show that $N k \times 2.2$ is necessary and sufficient to direct the development of Sim 1-expressing V3 INs (Briscoe et al., $1999,2000)$ and that the trophic effect of Nkx2.2 on V3 INs is antagonized by Olig2 (Sun et al., 2001). To assess whether this cross-repressive interaction requires a direct physical interaction between Nkx2.2 and Olig2 proteins, we conducted coelectroporation experiments as for Figure 7 above. Positive and negative controls are presented in Figure 8, $A$ and $B$. As shown, Nkx2.2 alone promotes ectopic expression of the Sim 1 marker for V3 INs (Fig. 8A), and this response is blocked by coelectroporation with full-length Olig2 (Fig. 8B). As noted also in our experiments with Cos7 cells (Fig. 3), we were unable to detect expression of a deletion fragment containing only the Olig2 bHLH domain in chick neural tube; however, combinatorial cotransfections show that an $\mathrm{N}$-terminal deletion of Olig2 has no effect on the cross-repressive interaction (Fig. $8 E$ ), whereas a C-terminal deletion has only a minor effect (Fig. $8 D$ ). Thus, the domains of Olig2 required for a physical interaction with Nkx2.2 are at least qualitatively sufficient for cross-inhibitory effects, in contrast to cooperative responses.

\section{Discussion}

The transcription factors Olig2 and Nkx2.2 belong to structurally distinct protein families (Chien et al., 1996; Harvey, 1996). As 
shown here, however, these two proteins recognize each other in antibody pulldown and yeast two-hybrid protocols. In each of these two conventional assays for detecting protein-protein interactions, the bHLH domain of Olig2 and the HD domain of $\mathrm{Nkx} 2.2$ appear necessary and sufficient for the interaction.

Additional evidence for an interaction between Olig2 and Nkx2.2 is obtained by monitoring the subcellular localization of Olig2 in Cos7 cells. The open reading frame of Olig2 does not encode a canonical nuclear localization signal (Lu et al., 2000). Deletion analysis (Fig. 5) shows that the Olig2 bHLH domain itself can function as a nuclear translocation signal. It would appear, however, that the bHLH domain is somehow masked in native Olig2 because the full-length protein is excluded from the nucleus in single transfection experiments (Fig. 5). Coexpression with a class $\mathrm{E}$ bHLH partner protein (E12) allows translocation of Olig2 to the nucleus (Fig. 4B); however, as shown, coexpression with Nkx2.2 will also relocalize Olig2 to the nucleus (Fig. 4C). In this relocalization assay, the NK2-specific domain of Nkx2.2 contributes to the interaction with Olig2. Therefore, as suggested schematically in Figure 9, a partner protein is required to unmask the nuclear localization signal (NLS) of Olig2 (Fig. 9).

Taken individually, the antibody pulldown experiments, the yeast two-hybrid studies, and the nuclear relocalization experiments must be interpreted with caution. Collectively, however, the results are internally consistent with each other and support the conclusion that Olig2 and Nkx2.2 interact to form a specific physical complex. Our data do not address whether the interaction is direct or indirect. In addition, we cannot quantitate the relative strength of this interaction for different deletion constructs. On a qualitative level, some of the interactions, for example that between full-length Olig2 and $\Delta \mathrm{Nkx} 2.2$ (112-273 aa), appear weaker than others. Within cells that express both transcription factors, it is conceivable that only a minor fraction of each protein participates in formation of the complex. Biological functions of the complex, if any, are unknown; however, we can gain some insight into functional relevance of the complex by combinatorial analysis of our deletion mutants in the developing chick neural tube. Our structure-function analysis is summarized in Table 2.

As indicated in Table 2, the domains required for the physical interaction are insufficient for, and perhaps irrelevant to, the cooperative interaction between Olig2 and Nkx2.2 that results in formation of Sox10-positive oligodendrocyte progenitor cells. This is perhaps not surprising. The bHLH and HD transcription factors have very distinct DNA recognition motifs. Conventional wisdom would suggest that their cooperative functions are ex- erted independently of each other via regulation of factor-specific gene sets; however, although the interaction between Olig2 and Nkx2.2 is not sufficient for oligodendrocyte development, our data do not preclude the possibility that the interaction is necessary for oligodendrocyte formation. To assess this possibility, we attempted to determine whether Nkx2.2 deletion mutations act in a dominant-negative manner to preclude development of endogenous oligodendrocytes in chick neural tube. None of our experiments (data not shown) suggested such a dominantnegative action; however, these negative results are ambiguous because endogenous oligodendrocytes appear at later times [embryonic day 5 (E5)-E6] in the ventral neural tube of the chick at a point where expression of ectopic genes from the CMV promoter used in our experimental system is much attenuated (see Materials and Methods).

We do not know how tightly Olig2 and Nkx2.2 bind to each other in vivo. Within cells that express both transcription factors at comparable levels, it is possible that only a minor fraction of 


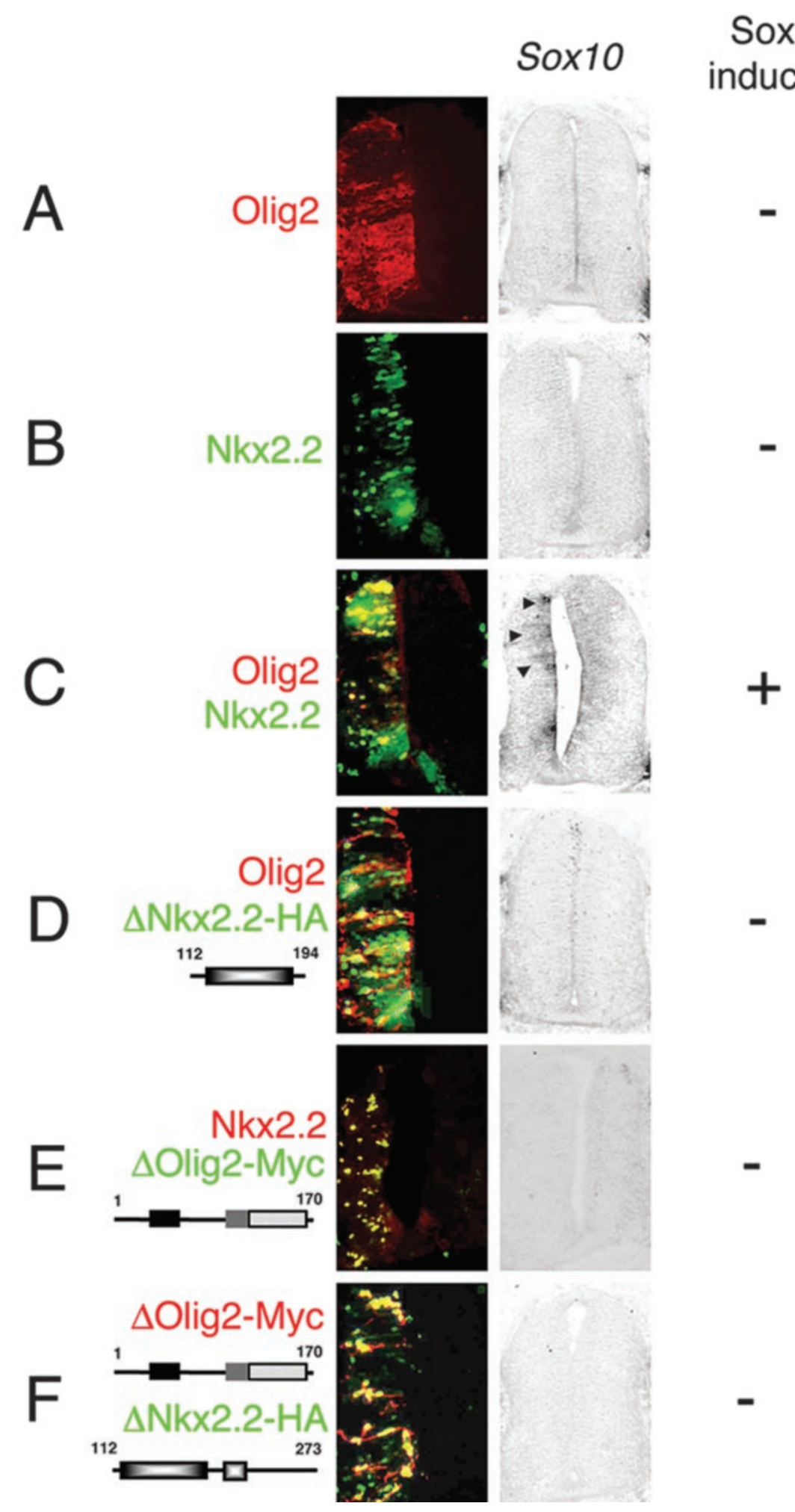

Figure 7. Interaction domains of Olig2 and Nkx2.2 are not sufficient for cooperative biological function. Expression constructs encoding full-length 0 lig2 (with Myc tag), Nkx2.2 (with HA tag) and their deletion mutations were electroporated into the neural tube of stage 10-12 chick embryos. At $48 \mathrm{hr}$ after electroporation, the embryos (stage 21-23) were harvested for analysis. Adjacent sections were immunolabeled with polyclonal or monoclonal antibodies against the 0 lig2 or Nkx2.2 expression products in red or green as indicated. Ectopic expression was directed to the left half of the neural tube, and the contralateral (non-electroporated) side served as a control. The embryos shown and the data summarized represent results from at least five analyzed embryos in all cases. Schemes of the 0 lig2 and Nkx2.2 expression constructs are shown to the left. The level of induction of (Sox10 as visualized by in situ hybridization is summarized by the + (induction) and the - (no induction) on the right. $A$, The ectopic expression of full-length Olig2 does not induce Sox10 expression. B, The ectopic expression of full-length Nkx2.2 does not induce Sox10 expression. C, Cooperative interaction: co-electroporation of Nkx2.2 and Olig2 induces Sox 10 expression. D, The homeodomain of Nkx2.2 is not sufficient for cooperative interaction with 0lig2. As shown, co-electroporation of the $\Delta N k \times 2.2$ (112-194 aa) with full-length Olig2 does not induce Sox10. E, Olig2 lacking the C terminus is not sufficient for cooperative interaction with Nkx2.2. As shown, co-electroporation of the $\Delta$ Olig2 (1-170 aa) with full-length Nkx2.2 does not induce Sox 10. F, Interaction of the homeodomain of Nkx2.2 and the bHLH domain of 0lig2 is not sufficient for the induction of Sox10. Co-electroporation of $\Delta$ Olig2 (1-170 aa) containing the bHLH domain and $\Delta N k \times 2.2$ (112-273 aa) lacking the $N$ terminus does not induce Sox10 expression. 
each transcription factor is involved in the physical complex that we describe here. Conversely, however, domains required for the protein-protein interaction overlap to a first approximation with domains required for cross-inhibitory effects in the chick neural tube assay (Table 2). This overlap might have functional overtones for establishment of a precise boundary between adjacent $\mathrm{p} 3$ and $\mathrm{pMN}$ progenitor domains during development.

Recent work has established that Olig2 expression defines the pMN and is necessary for motor neuron development in mice ( $\mathrm{Lu}$ et al., 2002; Takebayashi et al., 2002a; Zhou and Anderson, 2002). In the neural tube of $N k \times 2.2-/-$ embryos, the expression domain of the Olig2 extends ventrally and MN formation is augmented (Briscoe et al., 1999; Qi et al., 2001). Conversely, misexpression of $N k \times 2.2$ in the chick neural tube suppresses Olig2 expression in a cell-autonomous manner and inhibits motor neuron development (Novitch et al., 2001; Zhou et al., 2001); thus the cross-repressive action of $\mathrm{Nkx} 2.2$ may be mediated at the transcriptional level. In contrast, ectopic expression of Olig genes does not repress expression of $N k \times 2.2$ (Novitch et al., 2001). The data summarized in Figure 8 are consistent with the view that the cross-repressive action of Olig2 on Nkx2.2 is exerted at the posttranscriptional level via a physical interaction between the two proteins. In this scenario, the p3-pMN boundary formed within a narrow zone wherein Olig2 and Nkx2.2 are coexpressed. Within a few cells ventral to the developing boarder, transcription of Olig2 is suppressed by Nkx2.2 (present in relative excess). In a few cells dorsal to the border, formation of $\mathrm{V} 3$ interneurons is inhibited at the posttranscriptional level by entrapment of Nkx2.2 in a physical complex with Olig2 (also present in relative excess).

If a physical interaction with Olig2 antagonizes the ability of $\mathrm{Nkx} 2.2$ to induce $\mathrm{V} 3$ interneurons, it might be expected that the loss of Olig2 function (knock-out) would lead to an increase in V3 interneuron cell number; however, a dorsal expansion of V3 interneuron domain is not found in either Olig2 or Olig1/2 knock-out neural tube. Instead, a ventralization of the p2 domain, evidenced by ectopic high levels of Pax6 and Irx3 in the pMN, is observed (Lu et al., 2002; Zhou and Anderson, 2002). A cross-repressive interaction of Pax6 and Nkx2.2 has been shown previously by others (Ericson et al., 1997; Briscoe et al., 1999, 2000). It is likely therefore that ventralization of the Pax6 expression accounts for repression of Nkx2.2 expression and maintenance of the pMN-p3 border in Olig2-/- mice.

A post-transcriptional switching mechanism in neural cell fate

\section{$\operatorname{Sim} 1$}
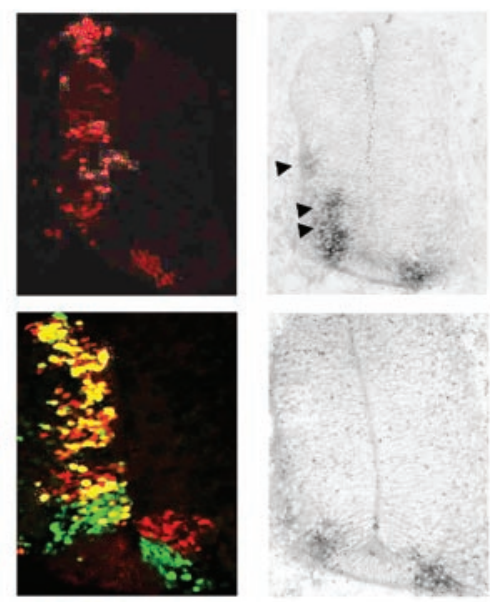

Nkx2.2
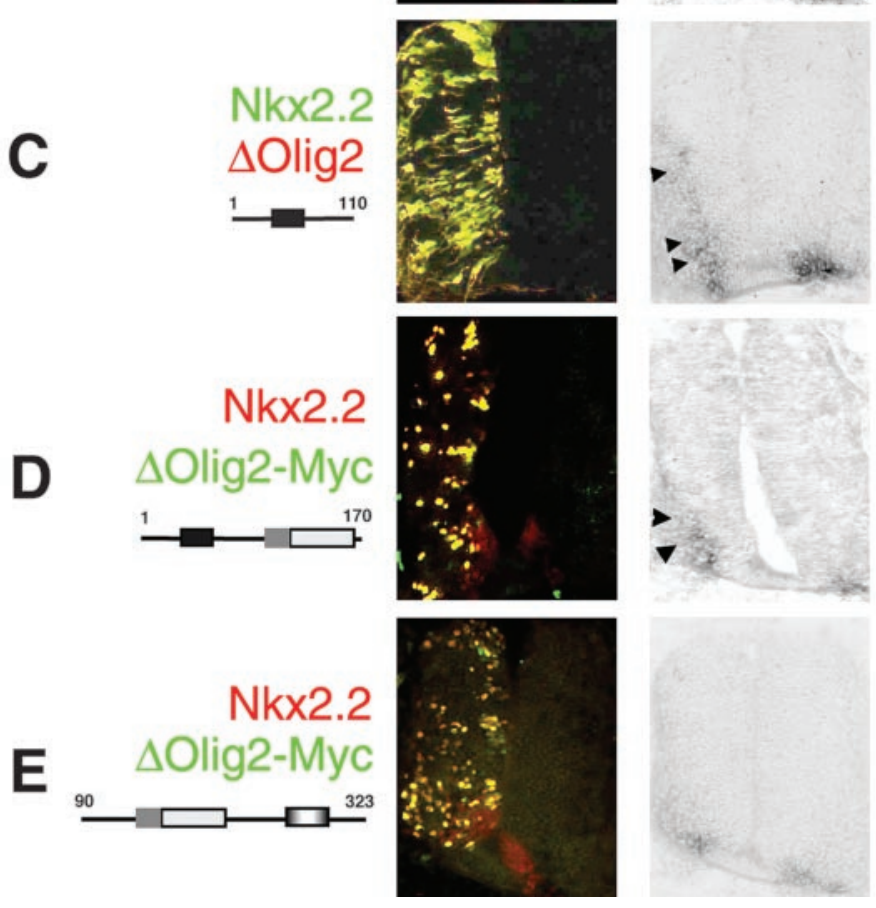

Nkx2.2 activity
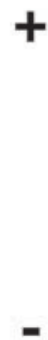

8. Interaction domains of 0 lig2 and Nkx2.2 are sufficient for cross-repressive function. Expression constructs encoding full-length Nkx2.2 (with Flag tag) and full-length 0lig2 and its mutations with Myc tag were co-electroporated into the neural tube of stage 10-12 chick embryos. After $48 \mathrm{hr}$, embryos (stage 21-23) were harvested for analysis. Adjacent sections were immunolabeled with polyclonal (red) or monoclonal (green) antibodies targeted to the Nkx2.2 or Olig constructs as indicated and hybridized in situ with a probe targeted to mRNA for chick cSim1. Ectopic expression was directed to the left half of the neural tube, and the contralateral (non-electroporated) side served as a control. Schemes of deletions of 0lig2 and Nkx2.2 with amino acid count are shown on the left. The level of induction of $\operatorname{Sim} 1$ as visualized by in situ hybridization is summarized by + (induction) or - (no induction) on the right. The embryos shown and results summarized are representative of at least five separate embryos in each case. $A$, The ectopic expression of full-length $N k x 2.2$ induces $\operatorname{Sim} 1$ expression. $B$, Cross-repressive interaction: co-electroporation of $\mathrm{Nkx2.2}$ and Olig2 inhibits induction of $\mathrm{Sim} 1$. C, Olig2 lacking the bHLH domain is not sufficient for cross-repressive interaction with Nkx2.2. As shown, co-electroporation of the $\Delta 0$ lig2 (1-110 aa) lacking the bHLH domain with full-length $N k \times 2.2$ does not inhibit Sim 1 induction by the Nkx2.2. D, 0lig2 fragment containing the amino terminal and bHLH domains is sufficient for cross-repressive interaction. Note however that loss of the C-terminal domain attenuates the repression somewhat. $E$, Olig2 fragment containing the $C$ terminal and bHLH domains is sufficient for cross-repressive interaction. choice has been noted in other contexts. For example, Lhx3 binds to the nuclear LIM interactor (NLI) to direct V2 interneuron formation. In motor neurons, however, Isl1 is available to compete for binding to NLI, displacing Lhx3 to a high-affinity binding site on the $\mathrm{C}$-terminal region of Isl1 and thereby transforming Lhx3 from an interneuron-promoting factor to a motor neuronpromoting factor (Thaler et al., 2002; Lee and Pfaff, 2003). This 


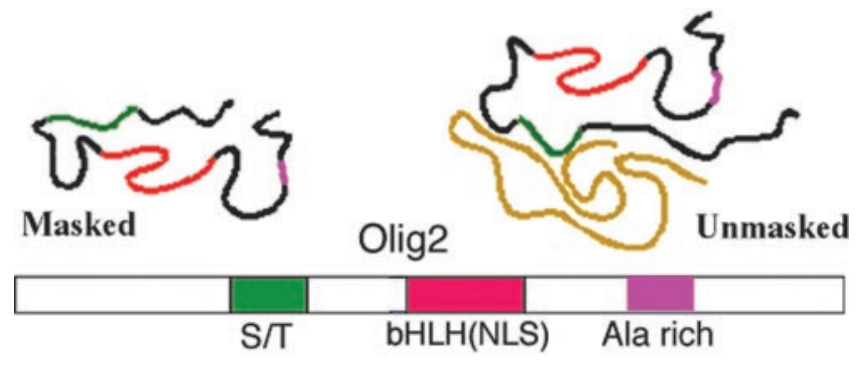

Partner Protein

Figure 9. An occluded NLS motif in Olig2. Our current model of 0lig2 proteins is shown. The bHLH domain, S/T domain, and Ala-rich domain are labeled separately. The model suggests that Olig2 has a functional NLS that may possibly be the bHLH motif itself. Olig2 monomers fold in such a way that the bHLH-NLS motif is occluded. The motif is unmasked by (1) interaction with bHLH partner proteins such as E12 (Fig. 4), (2) interaction with co-regulator proteins such as Nkx2.2 (Fig. 4), or deletion of flanking amino acids (Fig. 5).

Table 2. Summary of distinct regions of Olig2 and Nkx2.2 essential for nuclear localization, physical interaction, and cross-repression

\begin{tabular}{llll}
\hline Olig2 & S/T residues & bHLH domain & Ala-rich region \\
\hline Promoting MN formation & - & + & - \\
Physical interaction with Nkx2.2 & - & + & - \\
Cross-repression of Nkx2.2 & - & + & \pm \\
Induction of Sox10 & + & + & + \\
\hline Nkx2.2 & N terminus & Homeodomain & NK-2-specific domain \\
\hline Transporting Olig2 into nuclei & - & + & + \\
Physical interaction with 0lig2 & - & + & - \\
Induction of Sox10 & + & + & + \\
\hline
\end{tabular}

Nkx2.2 and 0lig2 protein structure analysis narrows down domains that are essential for their physical interaction and cross-repression in regulating neuronal subtype acquisition. The + indicates domains that are necessary for these interactions; the - indicates regions that are not essential for these interactions. The bHLH domain of Olig2 and the homeodomain of $\mathrm{Nk} \times 2.2$ are the core regions for their structural and functional interactions.

switching mechanism enables specific LIM complexes to form in each cell type and ensures that neuronal fates are tightly segregated.

Interactions of HD proteins and bHLH transcription factors have been demonstrated previously in pancreas and pituitary development and in muscle cell differentiation. The ubiquitously expressed bHLH protein E47 can form a complex with the homeodomain proteins Lmx1.1 or PDX-1 and activate Insulin transcription (Johnson et al., 1997; Ohneda et al., 2000). During pituitary development, the bHLH protein NeuroD1 is expressed in the corticotroph cells. Within these cells, NeuroD1 is dimerized with the ubiquitously expressed bHLH protein Pan1. This heterodimer forms a complex with the HD protein Pitxl and regulates target gene POMC expression (Poulin et al., 2000). In developing muscle, myogenic bHLH proteins form heterodimers with the pan-bHLH protein E2a and go on to interact with the HD protein Pbx1-Meis1/Prep (Knoepfler et al., 1999). Therefore regulation of target gene expression via interactions of HD proteins and bHLH transcription factors may be a general mechanism in multiple developmental processes.

\section{References}

Bertrand N, Castro DS, Guillemot F (2002) Proneural genes and the specification of neural cell types. Nat Rev Neurosci 3:517-530.

Briscoe J, Sussel L, Serup P, Hartigan-O'Connor D, Jessell TM, Rubenstein JL, Ericson J (1999) Homeobox gene Nkx2.2 and specification of neuronal identity by graded Sonic hedgehog signaling. Nature 398:622-627.

Briscoe J, Pierani A, Jessell TM, Ericson J (2000) A homeodomain protein code specifies progenitor cell identity and neuronal fate in the ventral neural tube. Cell 101:435-445.

Chien CT, Hsiao CD, Jan LY, Jan YN (1996) Neuronal type information encoded in the basic-helix-loop-helix domain of proneural genes. Proc Natl Acad Sci USA 93:13239-13244.

Ericson J, Rashbass P, Schedl A, Brenner-Morton S, Kawakami A, van Heyningen V, Jessell TM, Briscoe J (1997) Pax6 controls progenitor cell identity and neuronal fate in response to graded Shh signaling. Cell 90:169-180.

Gowan K, Helms AW, Hunsaker TL, Collisson T, Ebert PJ, Odom R, Johnson JE (2001) Crossinhibitory activities of Ngn1 and Math1 allow specification of distinct dorsal interneurons. Neuron 31:219-232.

Harvey RP (1996) NK-2 homeobox genes and heart development. Dev Biol 178:203-216.

Jessell TM (2000) Neuronal specification in the spinal cord: inductive signals and transcriptional codes. Nat Rev Genet 1:20-29.

Johnson JD, Zhang W, Rudnick A, Rutter WJ, German MS (1997) Transcriptional synergy between LIM-homeodomain proteins and basic helixloop-helix proteins: the LIM2 domain determines specificity. Mol Cell Biol 17:3488-3496.

Knoepfler PS, Bergstrom DA, Uetsuki T, Dac-Korytko I, Sun YH, Wright WE, Tapscott SJ, Kamps MP (1999) A conserved motif N-terminal to the DNA-binding domains of myogenic bHLH transcription factors mediates cooperative DNA binding with pbx-Meis1/Prep1. Nucleic Acids Res 27:3752-3761.

Lee JE, Hollenberg SM, Snider L, Turner DL, Lipnick N, Weintraub H (1995) Conversion of Xenopus ectoderm into neurons by NeuroD, a basic helixloop-helix protein. Science 268:836-844.

Lee SK, Pfaff SL (2003) Synchronization of neurogenesis and motor neuron specification by direct coupling of bHLH and homeodomain transcription factors. Neuron 38:731-745.

Lu QR, Yuk D, Alberta JA, Zhu Z, Pawlitzky I, Chan J, McMahon AP, Stiles CD, Rowitch DH (2000) Sonic hedgehog-regulated oligodendrocyte lineage genes encoding bHLH proteins in the mammalian central nervous system. Neuron 25:317-329.

Lu QR, Sun T, Zhu Z, Ma N, Garcia M, Stiles CD, Rowitch DH (2002) Common developmental requirement for Olig function indicates a motor neuron/oligodendrocyte connection. Cell 109:75-86.

Ma Q, Sommer L, Cserjesi P, Anderson DJ (1997) Mash1 and neurogenin1 expression patterns define complementary domains of neuroepithelium in the developing CNS and are correlated with regions expressing notch ligands. J Neurosci 17:3644-3652.

Mizuguchi R, Sugimori M, Takebayashi H, Kosako H, Nagao M, Yoshida S, Nabeshima Y, Shimamura K, Nakafuku M (2001) Combinatorial roles of olig2 and neurogenin2 in the coordinated induction of pan-neuronal and subtype-specific properties of motoneurons. Neuron 31:757-771.

Morgan BA, Fekete DM (1996) Manipulating gene expression with replication-competent retroviruses. Methods Cell Biol 51:185-218.

Murre C, McCaw PS, Vaessin H, Caudy M, Jan LY, Jan YN, Cabrera CV, Buskin JN, Hauschka SD, Lassar AB (1989) Interactions between heterologous helix-loop-helix proteins generate complexes that bind specifically to a common DNA sequence. Cell 58:537-544.

Novitch BG, Chen AI, Jessell TM (2001) Coordinate regulation of motor neuron subtype identity and pan-neuronal properties by the bHLH repressor Olig2. Neuron 31:773-789.

Ohneda K, Mirmira RG, Wang J, Johnson JD, German MS (2000) The homeodomain of PDX-1 mediates multiple protein-protein interactions in the formation of a transcriptional activation complex on the insulin promoter. Mol Cell Biol 20:900-911.

Park HC, Mehta A, Richardson JS, Appel B (2002) Olig2 is required for zebrafish primary motor neuron and oligodendrocyte development. Dev Biol 248:356-368.

Poulin G, Lebel M, Chamberland M, Paradis FW, Drouin J (2000) Specific protein-protein interaction between basic helix-loop-helix transcription factors and homeoproteins of the Pitx family. Mol Cell Biol 20:4826-4837.

Qi Y, Cai J, Wu Y, Wu R, Lee J, Fu H, Rao M, Sussel L, Rubenstein J, Qiu M (2001) Control of oligodendrocyte differentiation by the Nkx2.2 homeodomain transcription factor. Development 128:2723-2733.

Roztocil T, Matter-Sadzinski L, Alliod C, Ballivet M, Matter JM (1997) NeuroM, a neural helix-loop-helix transcription factor, defines a new transition stage in neurogenesis. Development 124:3263-3272. 
Sander M, Paydar S, Ericson J, Briscoe J, Berber E, German M, Jessell TM, Rubenstein JL (2000) Ventral neural patterning by Nkx homeobox genes: Nkx6.1 controls somatic motor neuron and ventral interneuron fates. Genes Dev 14:2134-2139.

Sun T, Echelard Y, Lu R, Yuk DI, Kaing S, Stiles CD, Rowitch DH (2001) Olig bHLH proteins interact with homeodomain proteins to regulate cell fate acquisition in progenitors of the ventral neural tube. Curr Biol 11:1413-1420.

Takebayashi H, Nabeshima Y, Yoshida S, Chisaka O, Ikenaka K (2002a) The basic helix-loop-helix factor olig2 is essential for the development of motoneuron and oligodendrocyte lineages. Curr Biol 12:1157-1163.

Takebayashi H, Ohtsuki T, Uchida T, Kawamoto S, Okubo K, Ikenaka K, Takeichi M, Chisaka O, Nabeshima Y (2002b) Non-overlapping expres- sion of Olig3 and Olig2 in the embryonic neural tube. Mech Dev 113:169-174.

Thaler JP, Lee SK, Jurata LW, Gill GN, Pfaff SL (2002) LIM factor Lhx3 contributes to the specification of motor neuron and interneuron identity through cell-type-specific protein-protein interactions. Cell 110:237-249.

Zhou Q, Anderson DJ (2002) The bHLH transcription factors OLIG2 and OLIG1 couple neuronal and glial subtype specification. Cell 109:61-73.

Zhou Q, Choi G, Anderson DJ (2001) The bHLH transcription factor Olig2 promotes oligodendrocyte differentiation in collaboration with $\mathrm{Nkx2.2}$. Neuron 31:791-807.

Zhuang Y, Barndt RJ, Pan L, Kelley R, Dai M (1998) Functional replacement of the mouse E2A gene with a human HEB cDNA. Mol Cell Biol $18: 3340-3349$. 